\title{
COMPARISON OF TWO HEMOSTATIC AGENTS IN PATIENTS RECEIVING ANTICOAGULANTS WITHOUT ALTERING MEDICATION DOSAGE
}

\author{
Hala R. Ragab* and Lydia N. Melek*
}

\begin{abstract}
Background: Patients under anticoagulant therapy who need tooth extraction, face the problem of intraoperative and postoperative bleeding which may be difficult to control. Some surgeons use the option of stopping the medication or altering its dose for three to four days preoperatively to obtain adequate hemostasis; however, this may carry the risk of thromboembolism.

Objective: This study aims to compare the effect of Gelatamp and Chitosan in control of postextraction bleeding and prevention of complications as dry socket in patients receiving anticoagulant therapy without altering the medication dose.
\end{abstract}

Materials and Methods: Patients were divided into 2 equal groups each consisting of 20 patients. Patients of group 1 underwent extraction followed by insertion of Gelatamp (gelatine sponge with colloidal silver). Patients of group 2 underwent extraction followed by insertion of Chitosan as a local hemostatic agent. All patients were examined postoperatively for bleeding after 5 minutes, 30 minutes, 2 hours and after 24 hours.

Results: Comparing the 2 groups in the different follow-up periods with regards to postoperative bleeding, a statistically significant difference was found between the Gelatamp and Chitosan groups at 5 minutes, 30 minutes and 2 hours post-extraction in favor of Gelatatmp, while the 2 groups showed a non-significant difference at 24 hours after extraction. At the time of suture removal, proper wound healing was found in patients of both groups with no signs of dry socket, inflammation or infection.

Conclusion: Both Gelatamp and Chitosan have provided adequate control of bleeding in patients receiving anticoagulants without altering drug dosage, with Gelatamp showing superior hemostatic effect in the immediate post-extraction period.

* Associate Professor of Oral and Maxillofacial Surgery, Faculty of Dentistry, Alexandria University 


\section{INTRODUCTION}

Warfarin is the most commonly used anticoagulant medication for patients with deep vein thrombosis, pulmonary embolism and prevention of stroke in patients with valvular heart disease, artificial valves and atrial fibrillation. It works as an antagonist for vitamin $\mathrm{K}$, the necessary vitamin for synthesis of coagulation factors II, VII, IX, X by the liver. ${ }^{(1)}$

Patients under anticoagulant therapy who need tooth extraction, face the problem of intraoperative and postoperative bleeding which may be difficult to control. If no sufficient hemostasis is achieved after oral surgery, complications as dry socket, delayed healing and pain may occur. Some surgeons use the option of stopping the medication or altering its dose for three to four days preoperatively to obtain adequate hemostasis; however, this may carry the risk of thromboembolism. ${ }^{(2)}$

In order to minimize bleeding complications and maintain the patient's drug dose unchanged, several protocols have been used including suturing of the wound, using pressure packs and application of various local hemostatic agents. ${ }^{(3)}$ Each of these techniques may be used individually or in combination to achieve the best control of bleeding and ensure proper wound healing.

Gelatamp is a completely resorbable hemostatic agent consisting of gelatine sponge with colloidal silver, which is used for prophylaxis of wound infections and control of post-extraction bleeding. Silver has a wide antibacterial effect over the resorption time of the material. Thus, Gelatamp provides rapid initial hemostasis, stability of the blood clot and a broad bactericidal effect. ${ }^{(4)}$

Chitosan has been approved for medical use in the United states in 2003. It is synthesized by deacetylation of chitin, which is the structural element in the exoskeleton of crustaceans (such as crabs and shrimp) and cell walls of fungi. Chitosan hemostatic agents are formed by mixing chitosan salts with an organic acid. The mechanism of action involves an interaction between the cell membrane of erythrocytes which is negatively charged and protonated chitosan which is positively charged leading to rapid clot formation. ${ }^{(5,6)}$

This study aims to compare the effect of Gelatamp and Chitosan in control of post-extraction bleeding and prevention of complications as dry socket in patients receiving anticoagulant therapy without altering the medication dose.

\section{MATERIALS AND METHODS}

This study is a randomized controlled clinical trial. It was conducted on 40 patients who were under anticoagulant therapy Coumarin derivatives and had unrestorable teeth indicated for extraction. Patients were operated upon in the Oral and Maxillofacial Surgery Department, Faculty of Dentistry, Alexandria University after giving an informed consent to approve their participation in the study. The research design was approved by the Institutional Research Ethics Committee.

Inclusion criteria were patients of both genders between 30 and 60 years of age, having an international normalized ratio (INR) within the therapeutic range $(\leq 3)$. Patients with comorbid hepatic, renal, hematological diseases or uncontrolled diabetes were excluded from the study.

All patients were assessed preoperatively and consultation with their physicians was done to confirm their medical status. They received their anticoagulant medication as usual without altering the dose.

Patients were divided into 2 equal groups each consisting of 20 patients. They were randomly allocated into each group using computer generated sequence.

Patients of group 1 underwent extraction followed by insertion of Gelatamp (gelatine sponge with colloidal silver, COLTENE/WHALEDENT Ltd., UK) into the socket. (Figure 1)

Patients of group 2 underwent extraction followed 
by insertion of Chitosan as a local hemostatic agent (Chitosan film high molecular weight HMW 2\% in $1 \%$ acetic acid provided by Sigma-Aldrich, USA and prepared by the Faculty of Pharmacy and Drug Manufacturing, Pharos University, Alexandria). (Figure 2)

Operative phase: all patients were administered local anesthesia with Mepivacaine 3\% without vasoconstrictor (Mepecaine, Alexandria Co., Egypt) followed by atraumatic extraction of the indicated tooth. The hemostatic agent (Gelatamp or Chitosan) was inserted into the socket and stabilized in place using figure of eight suture without tension. Patients were given the usual postoperative instructions.

Postoperative phase: All patients were examined postoperatively for bleeding after 5 minutes, 30 minutes, 2 hours and after 24 hours using the
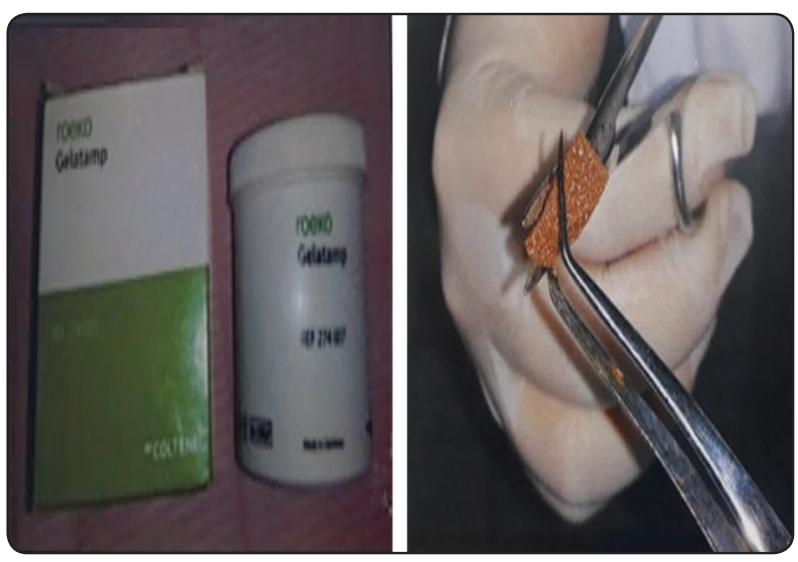

Fig. (1) Gelatamp

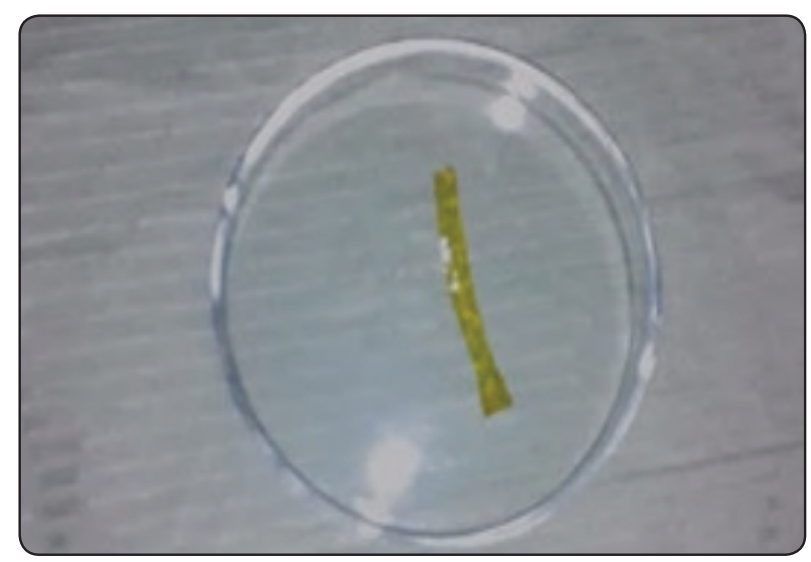

Fig. (2) Chitosan film following scale ${ }^{(7)}$ :

Grade 0: Very low (almost no bleeding).

Grade 1: Low (slight oozing of blood from the socket which usually stops by itself or after pressure is applied).

Grade 2: Normal. (Clinically significant).

Grade 3: High. (Bleeding occurs after clot has significantly formed).

Grade 4: Very high (excessive bleeding that could not be controlled by local hemostatic agents or stitches)

Pain was evaluated using numerical rating scale (NRS from zero to 10$)^{(8)}$ on the first, third and seventh days post-extraction.

Sutures were removed after one week and the extraction socket healing was evaluated clinically including assessment for the presence of any disintegrated blood clot, dry socket or signs and symptoms of inflammation/infection.

Data were collected and statistical analysis was done by descriptive statistics, Chi-square, Fischer's Exact and t-tests using Excel software (Microsoft Office 2016) and SPSS program version 23.0. A p-value $<0.05$ was considered statistically significant for all the comparisons.

\section{RESULTS}

The study was performed on 40 patients with a mean age of $50 \pm 7.23$ years. Group 1 included 8 females and 12 males while group 2 included 6 females and 14 males. The mean INR value in all patients was $2.5 \pm 0.5$.

Teeth extracted: The teeth extracted were upper anteriors (4 in group 1 and 5 in group 2), upper posteriors ( 7 in group 1 and 6 in group 2), lower anteriors (3 in group 1 and 4 in group 2) and lower posteriors (6 in group 1 and 5 in group 2). There was no statistically significant difference between the 2 groups regarding the type of tooth extracted $(\mathrm{p}=0.78)$. 


\section{Postoperative bleeding:}

Minor bleeding was observed post-extraction and insertion of the hemostatic agent in either group. Comparing the 2 groups in the different follow-up periods with regards to postoperative bleeding, a statistically significant difference was found between the Gelatamp and Chitosan groups at 5 minutes, 30 minutes and 2 hours post-extraction in favor of Gelatatmp, while the 2 groups showed a non-significant difference at 24 hours after extraction. (Table 1)

TABLE (1) Comparison between the 2 groups regarding the bleeding grade

\begin{tabular}{|c|c|c|c|c|c|c|c|c|}
\hline \multirow{2}{*}{$\begin{array}{c}\text { Bleeding } \\
\text { grade }\end{array}$} & \multicolumn{2}{|c|}{ After 5 minutes } & \multicolumn{2}{c|}{ After 30 minutes } & \multicolumn{2}{c|}{ After 2 hours } & \multicolumn{2}{c|}{ After 24 hours } \\
\cline { 2 - 10 } & Group 1 (n) & Group 2 (n) & Group 1 (n) & Group 2 (n) & Group 1 (n) & Group 2 (n) & Group 1 (n) & Group 2 (n) \\
\hline Grade 0 & 16 & 0 & 20 & 0 & 20 & 12 & 19 & 20 \\
\hline Grade 1 & 4 & 16 & 0 & 20 & 0 & 8 & 1 & 0 \\
\hline Grade 2 & 0 & 4 & 0 & 0 & 0 & 0 & 0 & 0 \\
\hline Grade 3 & 0 & 0 & 0 & 0 & 0 & 0 & 0 & 0 \\
\hline Grade 4 & 0 & 0 & 0 & 0 & 0 & 0 & 0 \\
\hline $\begin{array}{c}\text { Statistical } \\
\text { significance }\end{array}$ & $\begin{array}{c}\text { Pearson Chi-square= 27.20 } \\
\mathrm{p}-\mathrm{value}=0.00^{*}\end{array}$ & $\begin{array}{c}\text { Pearson Chi-square=40.00 } \\
\text { FE } \mathrm{p}=0.00^{*}\end{array}$ & $\begin{array}{c}\text { Pearson Chi-square=10.000 } \\
\text { FE } \mathrm{p}=0.003 *\end{array}$ & $\begin{array}{c}\text { Pearson Chi-square }=1.026 \\
\text { FE } \mathrm{p}=0.5\end{array}$ \\
\hline
\end{tabular}

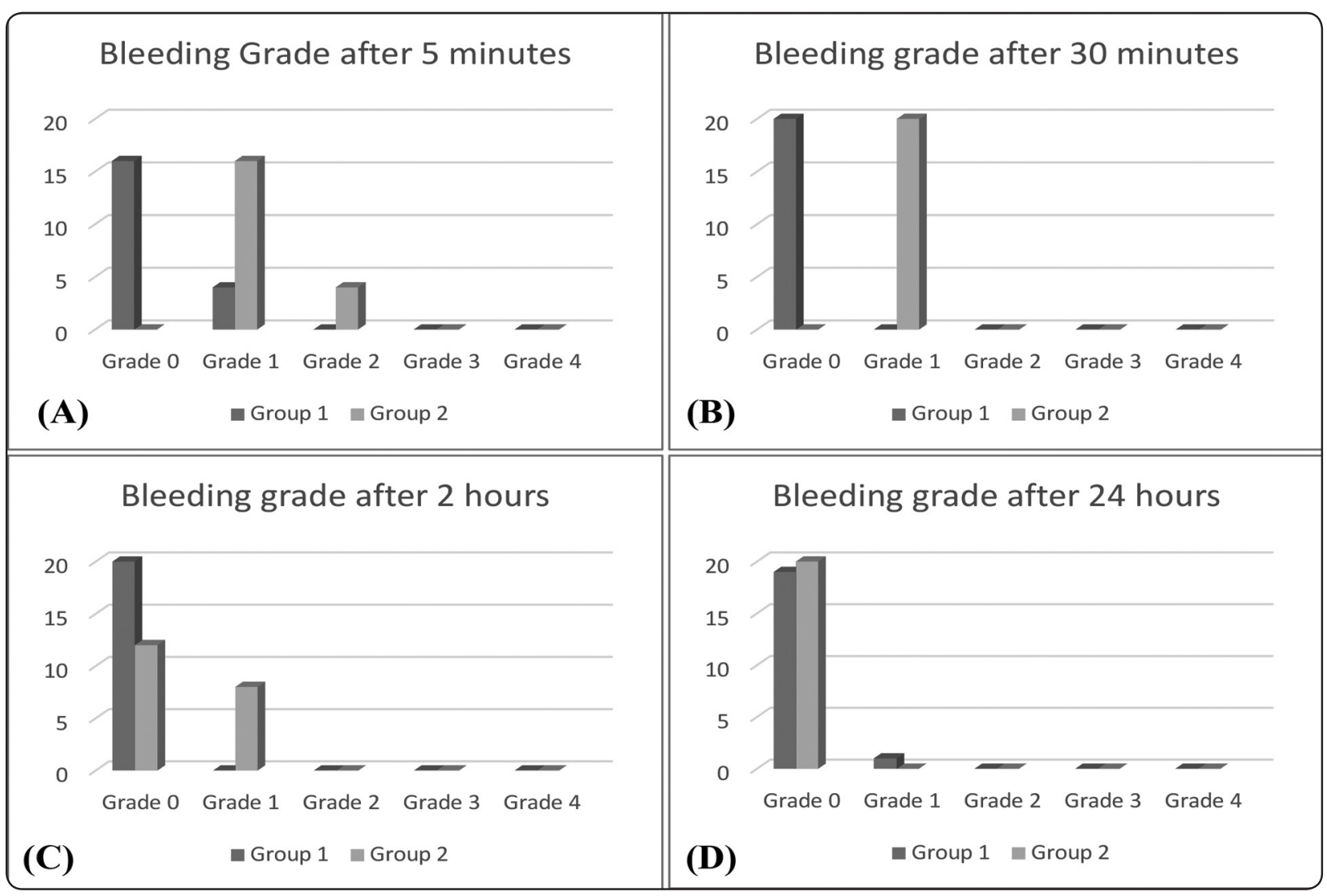

Fig. (3) Number of patients of the 2 groups represented in each of the Bleeding grades 


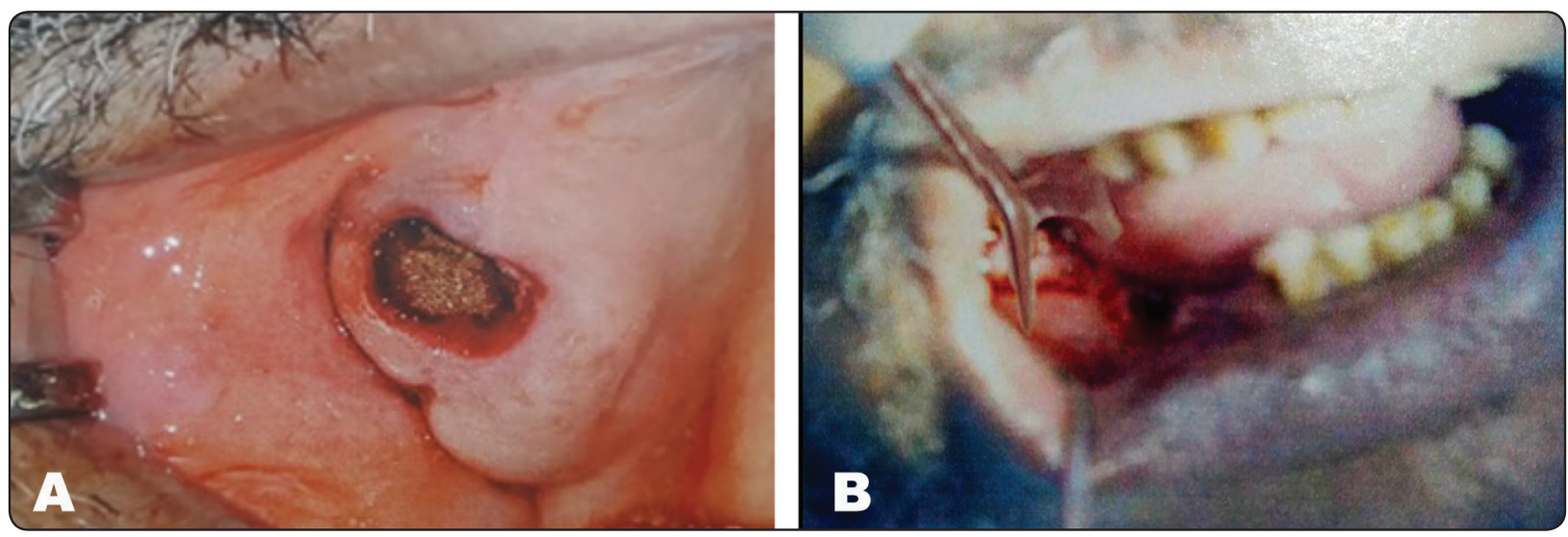

Fig. (4) A) Gelatamp delivery into the socket immediately after extraction. B) Chitosan film delivery into the socket immediately after extraction

Postoperative pain: The mean pain score for group 1 was $0.5 \pm 0.8$ and $0.25 \pm 0.47$ for group 2 on the first day with no statistically significant difference between both groups. It was completely resolved in all patients of both groups by the third postoperative day.

Wound healing: At the time of suture removal, proper wound healing was found in patients of both groups with no signs of dry socket, inflammation or infection.

\section{DISCUSSION}

Handling of patients with cardiovascular disease during dental surgical procedures may present a challenge to the oral surgeon depending on the degree of medical compromise and stability of their condition. In the last few decades, several protocols have been advocated for dealing with patients under anticoagulant therapy including withdrawal of the drug, reducing its dose, substitution with heparin, or continuation of the normal dose without interruption. ${ }^{(9)}$

Recently, more authors have proposed using local hemostatic measures for control of bleeding without interference with the anticoagulant drug regimen as a more conservative approach in order to avoid the risk of thromboembolism. ${ }^{(10)}$
Maani et al (7) have found that no significant difference in bleeding was observed between 2 groups, one in which the patients continued their medicine combined with the use of a local hemostatic agent and the other group in which patients stopped their medicine before extraction until INR reached 1.6. This precludes the need for anticoagulant suspension or reduction before minor oral surgical procedures where local hemostatic measures will be sufficient to control bleeding.

In accordance with that approach, we performed this study on patients receiving anticoagulant therapy without stopping or altering their medication dose before dental extraction (as was conventionally done in the past by suspension or dose reduction 3 to 4 days preoperatively). We divided patients into 2 equal groups and used two local hemostatic agents Gelatamp and Chitosan to control post-extraction bleeding comparing their hemostatic efficiency.

International Normalized Ratio (INR) is an international standard for the prothrombin time which is currently used as reliable measure for patients under oral anticoagulant therapy. ${ }^{(11)}$ In this study, patients of both groups were selected with INR within the therapeutic range $\leq 3$ as advised by Wahl. ${ }^{(12)}$ 
Chitosan was shown in previous studies to induce platelet adhesion and calcium mobilization resulting in reduction of the bleeding and clotting time. ${ }^{(13)}$ Also its biological properties have been investigated including local hemostasis, promotion of wound healing and antimicrobial effect, rendering it suitable for medical use. ${ }^{(14,15)}$

Hemostatic properties of Chitosan result from vasoconstriction, attraction of platelets, RBC's and clotting factors to the injury site. Also, it possesses natural antimicrobial characteristics due to the polycationic nature leading to disruption of gram-negative bacteria membranes. ${ }^{(16)}$ This was manifested in our study where the Chitosan group has shown adequate bleeding control and proper wound healing without adverse events.

Our results have indicated that the Gelatamp group exhibited significantly better hemostasis in the immediate postoperative period than the Chitosan group; however, no significant difference was found between the 2 groups after 24 hours. This goes hand in hand with the results of previous studies which declared the effectiveness of Gelatamp in bleeding control, where gelatin sponge provides a scaffold that facilitates clot formation. ${ }^{(7,17)}$

Gelatamp has both hemostatic and bactericidal properties in addition to complete resorption within 4 weeks. ${ }^{(17)}$ The small quantities of silver ions in Gelatamp provide the antimicrobial activity. ${ }^{(18)}$ This helps to achieve uneventful healing of the extraction socket without complications as encountered in the patients of our study, where no signs of infection, inflammation or dry socket were detected.

In the current study, both agents Gelatamp and Chitosan have provided adequate control of bleeding in patients receiving anticoagulants without altering drug dosage, with Gelatamp showing superior hemostatic effect in the immediate post-extraction period.

\section{CONCLUSION}

- Patients under anticoagulant therapy whose INR is within the therapeutic range need not stop or reduce their medication before dental extraction.

- The use of local hemostatic measures in such patients helps to control postoperative bleeding and achieve proper wound healing.

- Both Gelatamp and Chitosan have proved to be effective as local hemostatic agents with antimicrobial properties.

- Gelatamp has shown to provide better hemostasis in the immediate postoperative period.

\section{REFERENCES}

1. Hirsh J, Dalen J, Anderson DR, Poller L, Bussey H, Ansell $\mathrm{J}$, et al. Oral anticoagulants: mechanism of action, clinical effectiveness, and optimal therapeutic range. Chest. 2001;119(1 Suppl):8S-21S

2. Jaffer AK, Brotman DJ, Chukwumerije N. When patients on warfarin need surgery. Cleve Clin J Med. 2003; 70(11):973-84

3. Blinder D, Manor Y, Martinowitz U, Taicher S, Hashomer T. Dental extractions in patients maintained on continued oral anticoagulant: comparison of local hemostatic modalities. Oral surgery, oral medicine, oral pathology, oral radiology, and endodontics. 1999;88(2):137-40.

4. Gall RM, Witterick IJ, Shargill NS, Hawke M. Control of bleeding in endoscopic sinus surgery: use of a novel gelatinbased hemostatic agent. J Otolaryngol. 2002;31(5):271-4.

5. Zhang YJ, Gao B, Liu XW. Topical and effective hemostatic medicines in the battlefield. Int $\mathrm{J}$ Clin Exp Med. 2015;8(1):10-9.

6. Baldrick P. The safety of chitosan as a pharmaceutical excipient. Regul Toxicol Pharmacol. 2010;56(3):290-9.

7. Maani S SM, Melek L, Sadaka M. Evaluation Of Colloidal Silver Gelatin Sponge (Gelatamp) In Patients Receiving Anticoagulant After Tooth Extraction (Clinical Study). Alexandria Dental Journal. 2015;40:101-6.

8. Haefeli M, Elfering A. Pain assessment. Eur Spine J. 2006;15 Suppl 1:S17-24. 
9. Cannon PD, Dharmar VT. Minor oral surgical procedures in patients on oral anticoagulants-a controlled study. Aust Dent J. 2003;48(2):115-8.

10. Morimoto $\mathrm{Y}$, Niwa $\mathrm{H}$, Nakatani T. On the use of prothrombin complex concentrate in patients with coagulopathy requiring tooth extraction. Oral surgery, oral medicine, oral pathology, oral radiology, and endodontics. 2010;110(6):e7-10.

11. Pereira CM, Gasparetto PF, Carneiro DS, Correa ME, Souza CA. Tooth extraction in patients on oral anticoagulants: prospective study conducted in 108 brazilian patients. ISRN Dent. 2011;2011:203619.

12. Wahl MJ. Myths of dental surgery in patients receiving anticoagulant therapy. J Am Dent Assoc. 2000;131(1):7781.

13. Chou TC, Fu E, Wu CJ, Yeh JH. Chitosan enhances platelet adhesion and aggregation. Biochem Biophys Res Commun. 2003;302(3):480-3.
14. Muzzarelli RA. Chitins and chitosans as immunoadjuvants and non-allergenic drug carriers. Mar Drugs. 2010; $8(2): 292-312$.

15. Muzzarelli RA, Mattioli-Belmonte M, Pugnaloni A, Biagini G. Biochemistry, histology and clinical uses of chitins and chitosans in wound healing. EXS. 1999;87:251-64.

16. Kumar KR, Kumar J, Sarvagna J, Gadde P, Chikkaboriah S. Hemostasis and Post-operative Care of Oral Surgical Wounds by Hemcon Dental Dressing in Patients on Oral Anticoagulant Therapy: A Split Mouth Randomized Controlled Clinical Trial. Journal of clinical and diagnostic research : JCDR. 2016;10(9):Zc37-zc40.

17. Cai YH, Lu CS. [A clinical study of gelatamp colloidal silver gelatin sponge on preventing the complication of teeth extraction]. Hua Xi Kou Qiang Yi Xue Za Zhi. 2008; 26(5):519-21.

18. Tian J, Wong KK, Ho CM, Lok CN, Yu WY, Che CM, et al. Topical delivery of silver nanoparticles promotes wound healing. ChemMedChem. 2007;2(1):129-36. 